\title{
Review of B2B integration
}

\author{
Kenn Steger-Jensen and Hans-Henrik Hvolby \\ Manufacturing Information Systems Group (www.iprod.auc.dk/misg), Department of \\ Production, Aalborg University, Fibigerstraede 16, DK 9220 Denmark. \\ Email: \{kenn: hhh\}@iprod.auc.dk
}

\begin{abstract}
In this paper a review of $\mathrm{B} 2 \mathrm{~B}$ integration is presented based on non-proprietary standards. The focus is on technologies for back-office integration with suppliers and customers and, a recommendation to companies considering $\mathrm{B} 2 \mathrm{~B}$ integration.

Firstly, an introduction to $\mathrm{B} 2 \mathrm{~B}$ integration is presented by comparing underlying technology for B2B and EDI. Secondly, a framework for standardisation of B2B integration is presented organised in four tiers: Metadata tier, Content tier, Transport and Process tier. The framework is primarily based on contributions from standards and consortiums, and each tier is subsequently discussed. Furthermore, standardisation initiatives covering several tiers are presented; these are based on RosettaNet and ebXML. Finally, high-level scenarios with ebXML are described.
\end{abstract}

Keywords

B2B Integration, Enterprise Application Integration, and Application Integration Technology.

\section{INTRODUCTION}

During the last decade, B2B has obtained an extraordinary focus. One of the reasons is that most companies have already implemented ERP for internal/corporation use, and they are now ready to enter $\mathrm{B} 2 \mathrm{~B}, \mathrm{E}$-business and Supply Chain Management solutions. Another reason is that B2B evolution today has reached a level, which enables implementation of B2B solutions based on standards and technologies supported nearly by all major hardware and software vendors.

In connection with the Pathfinder project Gulledge and Sommer [1] found that baseline solutions for e-business and B2B were not as easy to establish as the different vendors' claim. One of the reasons was that many baseline solutions were not supported by other vendors' technologies and standards well enough. To obtain a satisfactory set-up, several baseline solutions had to be combined with customerspecific $\mathrm{B} 2 \mathrm{~B}$ programming.

Many B2B standards and flavours have risen during the last decade and the authors believe that many companies are not aware of the differences in these standards, which lead to expensive implementation cost for the companies. 
Normalisation has become the essential element in implementation of cost-effective B2B solutions. This paper will clarify the different B2B standards in order to review the validity of the standards and the functionality and technology which these standards support. This will be viewed in relation to implementation of costeffective $B 2 B$ solutions.

\section{B2B INTEGRATION}

Companies have always communicated with each other it is just the technology that carries this communication which has changed-from phone and mail to fax and EDI ... and now, to B2B communication via the Internet. Today, the Internet has changed the way business is conducted around the globe. The Internet enables companies to extend their market reach through new customer channels. Internet technologies provide an efficient, low cost media for business communication and sharing of data and, thus, enable multiple trading partners to collaborate on demand forecasts, production schedules and inventory requirements. This means that companies which leverage Internet business practices throughout their business processes - such as new product development, supply chain management, and procurement - can realise extensive benefits, as for instance shortened time to market, increased visibility across the extended supply chain, and reduced costs. Communication via the Internet, which is what B2B is when you look under the skin, is simply the latest communications technology to come along ... allowing communications to be more frequent, rapid and accurate.

First, B2B and EDI are compared before dealing further with B2B integration. Just like B2B, EDI allows electronic communication between businesses. However, there are two major differences. EDI is batch based while $B 2 B$ is real-time based, and $\mathrm{EDI}$ is dedicated point to point while $\mathrm{B} 2 \mathrm{~B}$ is more flexible.

XML has become the standard to define data interchange formats on the Internet. It is similar to HTML (hypertext mark-up language) in that it uses tags to encode information. But, whereas HTML tells browsers how to display information (focus on presentation), XML defines values for the information (focus on content, the data). XML also lets users create their own tags. XML is a way to enable data to have structure. The structure is not contained in the XML file itself but in a related text file (called a schema). A company who adopts the same structure for e.g. a Purchase Order can easily exchange orders via XML. The only problem is that multiple schemas, or multiple versions of descriptions for a Purchase Order have been created. Until schemas have been unified it is necessary to map the various schemas to each other to allow free and un-constrained communication.

To recognise and automatically use a flat file, which another business sends to you, is a major step forward. Computer systems are today rather advanced, but there is almost always some sort of manual intervention involved, leading to higher costs, time delays and errors. Therefore, B2B allows for communication, which is more frequent (real-time), rapid (on-line), and accurate (automated). 


\section{Framework for standardisation of B2B integration}

In this section each candidate in a framework for standardisation of B2B integration will be presented. Standardisation of B2B integration can be organised in four tiers: Metadata tier, Content tier, Transport and Process tier, which all must be covered for successful $\mathrm{B} 2 \mathrm{~B}$ integration. There are some pitfalls related to $\mathrm{B} 2 \mathrm{~B}$ integration which companies have to be aware of, because not all providers and standards of B2B integration components cover all tiers. At this time many different standards for each component of B2B integration have been established. This complexity in B2B standards makes it difficult for companies to select the right standards for their purpose.

- The Metadata tier describes data and how data are defined, with other words the syntax of the XML document. Focus is on representation of data in terms of document type definitions (DTDs) and schemas.

- The Content tier focuses on the XML-message and the way XML-messages are described. Often the description of e.g. a Purchase Order differs from company to company why standards for XML-messages are necessary. The primary candidates for XML-message standards are Open Applications Group and Collaborative Planning, Forecasting and Replenishment which both are supported by the main vendors of B2B and ERP systems.

* The process tier focuses on information flows in a company and between companies. Information flow is, in general, controlled by workflows, which in many cases are different from vendor to vendor. For example, workflow used in SAP R/3 is not the same as in ebXML or Navision. The primary candidate for process tier is the Workflow Management Coalition.

- The transport tier is necessary in order to exchange XML-messages from one ERP system to another. In some standards (ebXML, RosettaNet, Open Application Group) this component is often referred to as implementation framework. SOAP (Simple Object Access Protocol) is an XML-based protocol for exchange of structured and typed information in a decentralised, distributed environment.

Some standardisation initiatives cover several tiers, such as RosettaNet and ebXML are presented, as it is not possible to compare these multi-tier standard components with the single-tier standard components described in this section. In Figure 1, the different tiers and the main contributors are listed.

\begin{tabular}{|c|c|c|}
\hline \multicolumn{3}{|c|}{ Framework for XML-based B2B integration, and main contributors to the tiers } \\
\hline Metadata Tier & XML with Schemas or DTD, W3C & \\
\hline Content Tier & XML Messaging Model, OAG and CPFR & \\
\hline Process Tier & Workflow and business processes, WfMC & \\
\hline Transport Tier & SOAP XML-based protocol, W3C & \\
\hline
\end{tabular}

Figure 1 - Framework for B2B integration and main standard contributors to the tiers. 


\section{Metadata tier}

In this section the focus is on Metadata for XML, based on document type definitions and schemas, because both specify an abstract format for representation of data. XML is a data Nutshell. It is a generic data container, which also can reply to queries. The tool for querying and for transforming the data in an XML document is Extensible Stylesheet Language (XSL) [2], [3].

The W3C foresaw a need to describe the structure of XML documents to others. To do this, a simple set of Markup declarations were included in the language known as a DTD (document type definition). It can either be embedded within the instance document or, and more likely, referenced externally with an URL. A DTD defines an XML document's legal structure or grammar, which specifies which markup tags are available, where they may occur, and how they all fit together. A set of rules allows for XML parsers to validate a given document against its DTD, which reduces the needs for extensive exception checking in applications.

Before XML 1.0 was released as a W3C recommendation, it was obvious that a more rigorously definition of a XML document was necessary. In 1999 the XML schema working group was formed with exactly this purpose [4]. The group surveyed four proposals and came out with a schema recommendation in May 2001. Much like a DTD, an XML schema is a document, which describes the grammar of an instance document. It allows for more flexible and precise descriptions, and it is more up to date with related standards. Finally, it supports greater extensibility. The $\mathrm{XML}$ schema language lends XML the power and sophistication to create robust $\mathrm{XML}$ applications. By using this language it is possible efficiently and effectively to create and validate XML data. In a relational database, DDL is used to create tables and to specify rules and constraints for the tables. Similarly, the XML schema language provides the necessary framework for creating XML document's by specifying the valid structure, constraints, and data types for the various elements and attributes of the $\mathrm{XML}$ document. The schema language provides enhanced as well as more comprehensive and powerful features than DTD [2], [3].

The parser is the interface between an XML document and the application programme, which uses it. The XML processor is a software module, which reads XML documents and gives application programmes access to those documents' internal structure and content. The parser's main purpose is to pre-process XML documents and to provide application programmes with data, which is easier to operate with. The two most common types of XML parser are Document Object Model (DOM) and Simple Application programming interface for XML (SAX). The DOM parser converts an XML document to a tree structure (Stored in memory) before giving it to an application programme whereas a SAX parser generates events, which an application programme receives.

A XML file can be considered as a mini-database. The XML parser understands the database structure and can retrieve any set of data items or any specific data item. Data is retrieved from XML using XSL. XML excels in making on-line information search and retrieval fast and efficient. The reason is that XML documents also store Meta-information (like database). Search engines can use this feature to efficiently search and retrieve documents. 
One of the hottest application areas of XML is messaging. XML enables seamless and efficient transfer of data between applications. Because it is text based, all platforms can easily understand it. XML can be the perfect medium for exchange of information between organisations or within an organisation across different platforms. In the next section the contents of an XML-message are described and presented.

\section{Content tier}

In this section two candidates for the content tier are presented; OAG (Open Applications Group) and CPFR (Collaborative Planning, Forecasting and Replenishment). They both focus on the semantics of XML documents, which is the core of a content tier used for exchange of XML messages between business applications. As mentioned earlier, the key to implementation of cost-effective solutions is normalisation of B2B standards, which are made by or supported by $\mathrm{B} 2 \mathrm{~B}$ vendors. The problem is, in this case, that the vendors are more or less split into two camps beside the standard initiatives, which cover several tiers.

Authors find that The Open Applications Group may by the best choice between these two content tier vendors, because the majority of ERP systems are as default based on this model and has a good connection to ebXML. This is described in the following statement for Open Applications Group:

"The technical strategy of the Open Applications Group is to focus on the content of the Business Scenarios, the messages, and the dictionary, and to be technology sensitive to specific technical frameworks, but not technology specific. As a result of this strategy of technical neutrality, the Open Applications Group consciously decided not to build technical frameworks and other mechanisms that define how the business scenarios it builds are enabled and how the Business Object documents are transported. This gives the user the freedom to adopt the specific infrastructure that makes sense for their specific business use.

This strategy also perfectly positions the Open Applications work to be used with the ebXML technical work currently under development. The Open Applications Group plans to use the ebXML Transport work, the ebXML Business Process Collaboration Schema, and the ebXML Collaboration Partner Protocol (CPP) as the Collaboration Partner Agreement (CPA) specifications. The group plans to define or populate these bodies of work with their content definitions as soon as these documents are completed. The Open Applications Group is also working and watching the Registry and Repository work as well and watching and contributing to the Core Components work and will work for convergence with these bodies of work as it makes sense to their constituency",[5].

But, in the end it depends on the companies ERP system and the position/market shares and capability of standard initiatives, which cover several tiers (e.g. ebXML and RosettaNet). 


\section{The Open Applications Group}

The Open Applications Group, $[6,7,8]$, is a non-profit industry consortium. This group foresaw the rapidly growing need to integrate business applications fast and at low cost. This led to the development of Integration Specifications (OAGIS, [9]) which includes a broad set of XML schemas for sharing business information. It addresses the needs of traditional ERP integration as well as supply chain management and e-commerce. This specification provides the structure of business documents and additional meta-data, which is required as a part of the application processing.

The OAG has taken a conceptual approach to interoperability; a content-based virtual business object model, which enables an enterprise business application to build a virtual object wrapper around itself through the use of OAG compliant APIs. This model encapsulates the private implementation details of the object and enables communication through a public interface, which is shown in Figure 2. This interoperability is achieved with object-oriented advantages without the requirement to implement a software application with a specific object oriented technology.

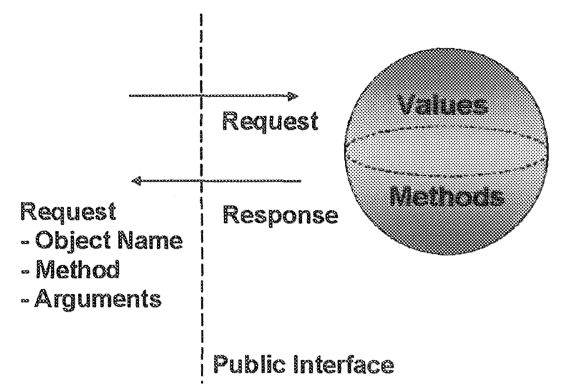

Figure 2 - The object model, which encapsulates the private implementation details of the object and enables communication through a public interface, [9].

The Business Object Document uses XML-schemas, a self-describing mechanism, to describe itself to other software components. The Business Object Document itself is not an object, it is an application architecture, which is used for conveying the communication and the necessary data to fulfil and carry out the requested business event. This is illustrated in Figure 3.

To communicate with a business software component in this model, events are communicated through the integration backbone in the form of an OAG compliant Business Object Document to a virtual object interface

The integration servers provide services such as publication and subscription, request and reply, transport mechanisms, data mapping tools, integration routing and logging capabilities. 


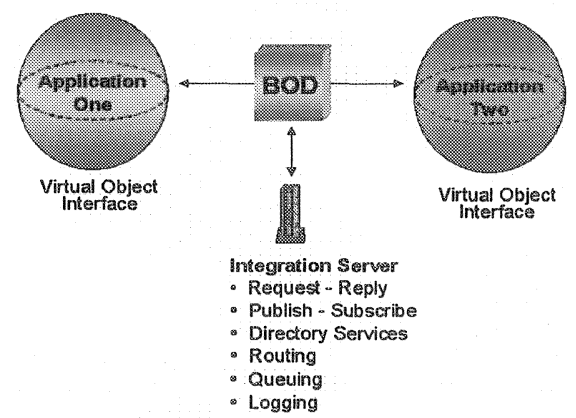

Figure 3 - The use of Business Object Document's (BOD), [9]

The Business Object Document is part of a larger model, which is fully described in the OAGIS, it is understood by the receiving application and can be processed accordingly. The Business Object Document is the application architecture used for execution of the virtual object interface. The Business Object Document contains the framework necessary to convey it into two primary components, the Control Area (Business Service Request) and the Business Data Area

The Business Service Request is the action which the Sender application wants the Receiver application to perform. The Business Data Area of the Business Object Document contains the codes, parameters and values needed to support the Business Service Request. For example, to send a Purchase Order to a business partner, the Business Data Area will contain the header and line information for all of the lines representing items to be purchased.

This Business Service Request and Business Data Area combination corresponds to the virtual business object models; Object Name, Method and Arguments, which are described in Figure 2. The business-object-document structure is presented in Figure 4. Business workflow (sequence) and exception handling support this common semantic model of communication.

The OAGIS is developed independently of the underlying technical framework used for routing and for delivering information. RosettaNet, ebXML and biztalk can exchange XML messages from OAG, which is already documented and published in other papers $[10,11,12]$. 


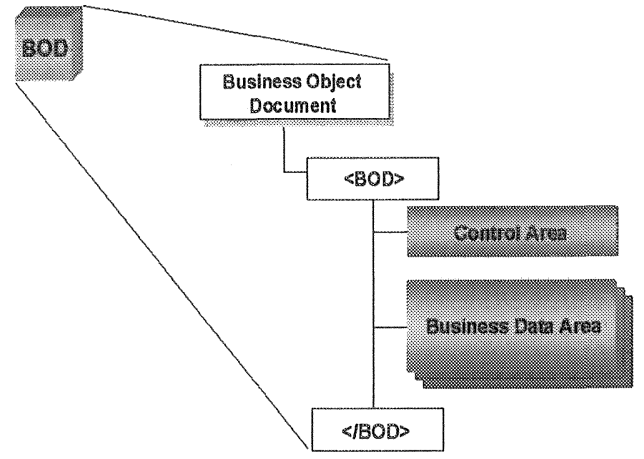

Figure 4 - The structure of the Business Object Document, and it two primary sub-areas, Control area and business data areas, [8].

\section{The Collaborative Planning, Forecasting and Replenishment Committee}

The Collaborative Planning, Forecasting and Replenishment (CPFR) [13, 14] Committee is a VICS committee made up of retailers, manufacturers, and solution providers. This group has developed a set of business processes, which entities in a supply chain can use for collaboration on a number of buyer/seller functions towards overall efficiency in the supply chain. The VICS CPFR XML specification has been integrated with the broader set of EAN.UCC XML specifications endorsed by the Global Commerce Initiative (GCI) to ensure full coverage of the CPFR process without creating overlapping or redundant message formats. The existing core EAN.UCC messages for item synchronisation, party (trading partner) synchronisation, purchase order, invoice, dispatch (shipment notice) and other information have been augmented with the CPFR product activity, forecast and other transactions.

Other XML-based industry standard efforts, such as RosettaNet and OAG have produced specifications for forecast and product activity data sharing. If companies who wish to deploy CPFR have investments in these transaction sets, they can use the RosettaNet or OAG transactions supplemented by the appropriate CPFR semantic of the XML message.

CPFR solutions may claim conformance to this specification. In many cases it is not appropriate to demand an "all or nothing" approach to conformance. For example, it is possible to deploy CPFR simply by sharing forecast and product activity data, and by allowing all exception criteria and exception processing to be handled locally. It is also possible that two installations may choose VICS EDI, RosettaNet, or OAG message formats to exchange forecast data, and at the same time use VICS CPFR XML message formats to share exceptions.

The CPFR Guidelines are, similar to OAGIS, transport independent. The CPFR XML Messaging model does not include message headers describing transport or routing mechanisms, which is the core of the transport tier and SOAP. Several other initiatives, such as RosettaNet, BizTalk, OBI and ebXML include XML-based transport and routing specifications. CPFR implementations can make use of any of these (including transports unrelated to XML) for message exchange. 


\section{Process tier}

In this section a process tier candidate, Workflow Management Coalition (WfMC), is presented. There are others, but the reason for choosing this candidate is that a strong relationship to ebXML work exists, which is explained later in this section.

The Workflow Management Coalition (WfMC) $[15,16,17]$ has released a Workflow Standard - Interoperability: Wf-XML Binding version 1.1, which is a specification of a language based on the XML and designed to model the data transfer requirements set forth in the specification of WfMC's Interoperability Abstract.

This specification is based on previous work completed by the WfMC, the Object Management Group (OMG) and many vendor organisations in an effort to define the functionality required to achieve interoperability among workflow systems. With this release, the WfMC has further defined the fundamental protocol elements required to support interoperability among workflow systems over any transport mechanism (such as HTTP, SMTP, TCP/IP, etc.), whether synchronous or asynchronous.

A great deal of commonality can be found between ebXML Requirements Specification [18] and the WfMC Workflow Reference Model [19]. The WfMC process definition attempts to provide a generic capability, which is equally applicable for internal processes within one organisation, or for a process which spans several organisations. The other underpinning principle behind the WfMC work is that the process definition can be encoded in a machine interpretable form to support automation on a different platform to the definition work (i.e. it is as much on interchange specification as a modelling approach). The (Wf-XML) specification defines the runtime interactions between process automation systems to support process enactment across multiple domains (either within or between organisations). WfMC has not attempted to address the wider e-commerce contract management issues other than with guidance on interoperability contracts between organisations. A lot of initiatives take place in this area of the ebXML work, and it appears to be necessary in order to get it working in real life, as mentioned earlier the problem is again lack of normalisation.

\section{Transport tier}

In this section a Transport tier candidate, Simple Object Access Protocol (SOAP), is presented. SOAP is a lightweight XML-based protocol for exchange of structured and typed information, which defines the XML formats of B2B interaction of requests and responses. SOAP is a specification for invoking methods and objects, and it codifies the exiting practice of using XML and HTTP as a method-invoking mechanism.

The motivation for development of SOAP was that existing Remote Procedure Calls protocols as DCOM (Microsoft's distributed component model protocol), CORBA's remote invocation protocol called Internet Inter-ORB (CORBA-IIOP) or J2EE RMI (Remote Method Invocation), both were designed for use in LAN and eventually they were either blocked by the firewall and proxy servers or they were 
dependent on platform respective program language. Distributed applications, which are constipated to communicate with many different partners over Internet, cannot grab to such protocols. SOAP is typically used in connection with firewall friendly transports such as SMTP or HTTP, although other transports such as FTP can be used.

The SOAP XML-based, COM-like protocol simplifies information exchange across a variety of platforms because it is not tied to any specific object model. For example, a client written in Microsoft Visual Basic could use SOAP to access a method in a Common Object Request Broker Architecture (CORBA) object running on a Unix platform [20]. Both CORBA's IIOP and SOAP provide ways of marshalling and un-marshalling arguments - in SOAP's case the underlying marshalling language is XML, which is platform independent. Exactly this property and characteristic is what makes SOAP interesting. Before SOAP was developed it was difficult to access a supplier's ERP system running on a platform and call a method in their system.

The benefits of SOAP as part of the B2B integration definition are that SOAP also provides a way to leverage the huge industry investment in XML. Moreover, since SOAP is typically defined via the firewall friendly protocols such as HTTP and SMTP, the huge industry investment in firewall technology is leveraged as well. The result is the elimination of major barriers to $\mathrm{B} 2 \mathrm{~B}$ integration.

A SOAP message consists of a SOAP envelope which encloses two data structures, the SOAP header and the SOAP body, [21]. The envelope contains, in addition to header and body, information defining the name spaces used in the definition of the enclosed data structures. The header is a generic mechanism for adding features to a SOAP message without prior agreement between the communication parties. The header is optional, but when present it is intended to convey information about the request defined in the SOAP body. For example, it might contain transactional, security, contextual or user profile information.

The body contains an XML massage request or reply to a request in XML format, and it is a container for mandatory information intended for the ultimate recipient of the message. It defines which object and method is addressed and which parameters are transferred.

SOAP message exchange can be modelled as Remote Procedure Calls (RPCs), with a well-defined way of message exchange based on well-defined method call signatures (that is, method name and parameters) and its return values/exceptions. SOAP messages can also be viewed as documents exchanged where the semantics of the data exchanged are known only at the application level (sender or receiver).

\section{Summary}

B2B integration is heavily dependent and based on XML and primarily with XML schemas from the Metadata tier, which gives the capability to exchange XMLmassages, the core of the content tier. The process tier is a core element according to business processes and interaction based on workflow. The WfMC process definition attempts to provide a generic capability for internal processes within one organisation, or for a process, which spans several organisations. A typical B2B 
integration solution is the discrete system model, in this example between OAGI and RosettaNet, which is as follows:

$$
\text { ERP(OAGI) - B2B(RNIF) - . - B2B(RNIF) - ERP(OAGI) }
$$

Unfortunately, a one-to-one relationship does not exist between OAGI and RosettaNet messages, or for that matter between other content tier vendors and vendors who cover several tiers.

The majorities of ERP vendors are part of OAGI, and the majority of ERP systems are based on OAGI messages. At the same time, all XML-message vendors and standardisation initiatives cover several tiers such as RosettaNet, and ebXML has its own workflow and exception handling method related to the XML-message. Therefore, if the contribution from WfMC becomes a success, mapping between different workflow systems and different $\mathrm{B} 2 \mathrm{~B}$ solutions will be reduced drastically.

It is not only the workflow and XML-message, which have to be mapped, but also the exception handling method. If two companies have implemented different $\mathrm{B} 2 \mathrm{~B}$ solutions, mapping between these two solutions could be necessary. On the transport tier SOAP and HTTP are the backbone. SOAP will presumably in future be the primary XML-message format, because it supports the connection between businesses in general and allows sending and receiving a document and RPC and, furthermore, it is firewall friendly. The majority of standardisation initiatives, which covers several tiers have explicitly defined a mechanism for secure exchange of XML messages via an HTTP based infrastructure using the Internet standard MIMEbased encoding and related technologies, such as SOAP.

\section{STANDARD INITIATIVES COVERING SEVERAL TIERS}

In this section, two initiatives covering several tiers will be presented. Firstly, RosettaNet will be introduced and, secondly, ebXML. A high-level case scenario for two Trading Partners with ebXML will be described.

\section{RosettaNet}

RosettaNet, [22, 23] is an independent, self-funded, non-profit consortium of major Information Technology (IT), Electronic Components (EC) and Semiconductor Manufacturing (SM) vendors. Dedicated to the development and deployment of open electronic commerce standards which align the business processes between partners in high-tech supply chains, RosettaNet specifications allow manufacturers, distributors, resellers and end-users to leverage the Internet to exchange business documents across the entire supply chain on a global basis. RosettaNet standardisation efforts can be divided into three broad groups of data format, business process and protocol specification:

- RosettaNet Dictionaries

- RosettaNet PIPs (Partner Interface Processes)

- RosettaNet Implementation Framework 


\section{RosettaNet Dictionaries}

The RosettaNet dictionaries, [24], define a common set of properties for use by the business process (PIP) specifications and associated business documents and guidelines. RosettaNet dictionaries are classified as Business and Technical Dictionaries.

- The RosettaNet Business Dictionary defines the properties used in basic business activities between trading partners. These are the Business Properties (e.g. business address), Business Data Entities (e.g. ActionIdentity) and Fundamental Business Data Entities (e.g. businessTaxIdentifier, AccountNumber) etc. Only one business dictionary encompasses all supply chains (EC, IT etc).

- The RosettaNet Technical Dictionary defines properties for products, components/devices and services that span the EC, IT and other supply chains.

\section{RosettaNet PIPS}

RosettaNet Partner Interface Processes ${ }^{\mathrm{TM}}$ (PIPs ${ }^{\mathrm{TM}}$ ) [25] define the specific sequence of steps required to execute business processes between supply chain partners. This is also known as the business process choreography.

Examples of such processes include purchase order management and distribution of new product information. In addition to the business process choreography, a RosettaNet PIP specification includes the specification of structure and content format of the business documents exchanged (XML DTDs and guidelines) and the time, security, authentication and performance constraints on these interactions.

Specification of the PIPs is limited to publicly visible interactions and interfaces between trading partners. Hence, these are also referred to as the public processes. In contrast, private processes are business processes within an enterprise, which trigger the execution or are triggered by the execution of the public processes or the PIPs. RosettaNet divides the entire e-business supply chain domain for which PIPs are specified into broad classifications called clusters. Each cluster is further subdivided into two or more segments. Each segment comprises several PIPs. PIPs contain one or more Activities, and Activities in turn specify Actions. An example of this relationship follows, [25]:

* CLUSTER 3: Order Management

a Segment A: Quote and Order Entry

a PIP 3A4: Manage Purchase Order

- Activity: Request Purchase Order

\section{RosettaNet Implementation Framework}

The RosettaNet Implementation Framework (RNIF) $[26,27]$ provides the fundamental plumbing required to execute business processes between trading partners in an open, interoperable, secure and independent way regarding platforms and implementation. The RNIF explicitly defines a mechanism for secure exchange of XML messages over an HTTP based infrastructure using the Internet standard 
MIME-based encoding and related technologies (See the RNIF specification for complete references).

Therefore, RosettaNet supports dictionaries for search of information on a business classified as Business and Technical Dictionaries. PIPs support business processes which are the process tier in the framework, and RNIF supports the Transport tier in the framework mentioned earlier. All this B2B integration is based on XML DTD (Metadata tier), and on XML-messages (Content tier). Some XML DTDs are already replaced by XML schemas instead but not all, jet.

\section{ebXML}

ebXML (electronic business with XML) [28] is a complete B2B framework which enables business collaboration through the sharing of Web-based business services. It is an effort of UN/CEFACT and OASIS. UN/CEFACT is the United Nations Centre for Trade Facilitation and Electronic Business, and OASIS is, an Organisation for the Advancement of Structured Information Standards, a not-forprofit, global consortium that drives the development, convergence and adoption of e-business standards.

The first version was finished in May 2001. The framework supports the definition and execution of $\mathrm{B} 2 \mathrm{~B}$ processes expressed as choreographed sequences of business service exchanges. The framework includes specification for a Message service, Collaborative Partner Agreements (CPA), Core Components, Business Process Methodology, and Registry and Repository. Technical committees for the ebXML Registry, Messaging, Collaborative Partner, and Implementation are hosted by OASIS, and Business Process and Core Component work continues at UN/CEFACT. The conceptual overview described below introduces the following concepts and underlying architecture:

The ebXML Registry Service serves as the storage facility for the Business Process and Information Models, the XML-based representations of those models, Core Components, and Collaboration Protocol Profiles. The ebXML Business Process and Information Meta Model supports requirements, analysis and design viewpoints that provide a set of semantics for each viewpoint and forms the basis of specification of the artefacts that are required to facilitate Business Process and information integration and interoperability. Business Processes are created by applying the UN/CEFACT Modelling Methodology (UMM) which utilises a common set of Business Information Objects and Core Components. A Core Component captures information about a real world business concept, and the relationships between that concept, other Business Information Objects, and a contextual description that describes how a Core or Aggregate Information Entity may be used in a particular ebXML eBusiness scenario. A Business Process document refer to the Core Components directly or indirectly using a XML document that references the appropriate Business and Information Models and/or Business Documents (possibly DTD's or Schemas).

An additional view of the Meta Model, the Specification Schema, is also provided to support the direct specification of the set of elements required to configure a runtime system in order to execute a set of ebXML business transactions. By drawing out modelling elements from several of the other views, 
the Specification Schema forms a semantic subset of the ebXML Business Process and Information Meta Model. The Specification Schema is available in two standalone representations, a UML profile, and a DTD. The Specification Schema supports the specification of business transactions and the choreography of business transactions into Business Collaborations. Each Business Transaction can be implemented using one of many available standard patterns. These patterns determine the actual exchange of Messages and signals between Trading Partners to achieve the required electronic transaction. To help specify the patterns the Specification Schema is accompanied by a set of standard patterns and a set of modelling elements common to those patterns. The full specification of a Business Process consists of a Business Process and Information Meta Model specified against the Specification Schema and an identification of the desired pattern(s). This information serves as the primary input for the formation of Collaboration Protocol Profiles (CPP's) and Collaboration Protocol Agreements (CPA's).

\section{Collaboration Protocol Profiles and Collaboration Protocol Agreements}

To facilitate the process of conducting eBusiness, potential Trading Partners need a mechanism to publish information about the Business Processes they support along with specific technology implementation details about their capabilities for exchanging business information. This is accomplished through the use of a CPP. The CPP is a document which allows a Trading Partner to express their supported Business Processes and Business Service Interface requirements in a manner where they can be universally understood by other ebXML compliant Trading Partners.

A special business agreement called a CPA is derived from the intersection of two or more CPP's. The CPA is a document that represents the intersection of two CPP's and is mutually agreed upon by both Trading Partners who wish to conduct eBusiness using ebXML. A CPA describes: (1) the Messaging Service and (2) the Business Process requirements that are agreed upon by two or more Trading Partners. A CPA contains the Messaging Service Interface requirements as well as the implementation details connected with the mutual Business Processes that both Trading Partners agree to use to conduct eBusiness. Trading Partners may decide to register their CPA's in an ebXML compliant Registry Service, but this is not a mandatory part of the CPA creation process.

\section{ebXML business process example}

The business process between two companies with ebXML is as follows: Company A has become aware of an ebXML Registry that is accessible on the Internet. After reviewing the contents of the ebXML Registry it decides to build and deploy its own ebXML compliant application. Custom software development is not a necessary prerequisite for ebXML participation. ebXML compliant applications and components may also be commercially available as shrink-wrapped solutions. The Business Profile information (including implementation details and reference links) is submitted to the ebXML Registry. The business profile submitted to the ebXML Registry describes the company's ebXML capabilities and constraints, as well as its supported business scenarios. These business scenarios are XML versions of the Business Processes and associated information bundles in which the company 
is able to engage. After receiving verification that the format and usage of a business scenario is correct, an acknowledgement is sent to Company A.

Company $\mathrm{B}$ discovers the business scenarios supported by Company $\mathrm{A}$ in the ebXML Registry. Company B sends a request to Company A stating that they would like to engage in a business scenario using ebXML. Company $B$ acquires an ebXML compliant shrink-wrapped application. Before engaging in the scenario, Company B submits a proposed business arrangement directly to Company A's ebXML compliant software Interface. The proposed business arrangement outlines the mutually agreed upon business scenarios and specific agreements. The business arrangement also contains information pertaining to the messaging requirements for transactions to take place, contingency plans, and security-related requirement. Company $\mathrm{A}$ then accepts the business agreement.

Company $\mathrm{A}$ and $\mathrm{B}$ are now ready to engage in eBusiness using ebXML.

\section{CONCLUSIONS}

A framework and the main candidates in each tier of the framework for standardisation of B2B integration have been presented. Each candidate has their own way of communicating and some candidates even cover several tiers.

The primary problems in implementing cost-effective solutions today are related to the mapping between different standards, and the fact that not all XML-schemas are well supported by all XML-parsers.

Communication via the Internet using e.g. SOAP as an XML protocol contains a XML massages request or replies to a request in XML format. A lot of standardisation initiatives offer a semantic for the XML-messages, but no one-to-one relationships exist between the different XML-messages.

ebXML is already implemented in many businesses and is a non-profit industry consortium comprised of many of the most prominent stakeholders in the business software component industry throughout the world, this will push further developments. Therefore, ebXML probably is the best candidate for B2B integration as it covers all tiers.

\section{REFERENCES}

[1] Links to all web-references and the PIP 3A4 standard are placed at our homepage, http://www.iprod.auc.dk/misg/papers/apms2002.htm

[2] Gullege, R. Thomas and Sommer, A. Ray, Integration ebusiness Transaction Across Extended Enterprises, Proceedings of the IFIP 5.7 International Working Conference on Strategic Manufacturing, 26-29 August 2001, Aalborg, Denmark, pp. 177 - 189.

[3] J Roy and A Ramanujan, XML: Data's Universal Language, IT Professional, 2000, May-June, pp. 32 - 36 .

[4] J Roy and A Ramanujan, XML Schema Language: Taking XML to the Next level, IT Professional, 2001, March-April, pp. 37 - 40.

[5] http: //www.w3.org/XML/Schema 
[6] Open Applications Group adoption of ebXML, David Connelly, President and CEO Open Application Group, Inc. http://www.gca.org/papers/xmleurope2001/papers/pdf/s18-2e.pdf

[7] Open Applications Group, Inc., Plug and Play Business Software Integration, The Compelling Value of the Open Applications Group,http: //www.openapplications.org/

[8] Open Applications Group, Inc., OAGIS Extensions, Exploring Extensions, Open Applications Group, Incorporated Document Number 20010301, Copyright (C) 2001 http: //www.openapplications.org/

[9] Open Applications Group, Incorporated, Release 1.0, Document Number OAMAS_990815, Open Applications Group Common Middleware API Specification. Copyright $\odot 1999-2000$. http: //www.openapplications.org/

[10] Open Applications Group, Inc., OAGIS Release 7.2.1 http: //www.openapplications.org/

[11] The Open Applications Group and RosettaNet, Implementing OAGIS within the RosettaNet Implementation Framework Version 2.0, Last Modified: 2/21/2001, Kurt Kanaskie, Lucent Technologies, Inc., 2000, http: //www.openapplications.org/

[12] OAGIS Implementation Using the ebXML CPP, CPA and BPSS specifications v1.0, Jean-Jacques Dubray, eXcelon Corp. VERSION 1.04, LAST MODIFIED: 10/4/2001 http: //www.openapplications.org/

[13] BizTalk and the Open Applications Group, Implementing OAGIS within the Microsoft BizTalk Framework, Christopher Kurt, 14 June 2000. http: //www.openapplications.org/

[14] VICS CPFR ${ }^{\circledR}$ XML Messaging Model, June 25, 2001 Voluntary Interindustry Commerce Standards Association Copyright () 2001. http: //www.cpfr.org/

[15] Collaborative Planning Forecasting and Replenishment Voluntary Guidelines. http://www.cpfr.org/

[16] The Workflow Management Coalition Specification, Workflow Management Coalition Workflow Standard Interoperability Wf-XML Binding, Document Number WFMC-TC1023, Document Status: Final Draft. 14 - November - 2001, Version 1.1, Copyright 1999 - 2001 The Workflow Management Coalition. http: //www.wfmc.org/

[17] Workflow Standard Interoperability, XML-HTTP binding, Document Status: Submission 2.0 8 February, 2000, Nobuyuki Kanaya, Shinichi Iwasaki, Akihiko Suzuki, Tsuyoshi Ebata, Hirotaka Hara. Information technology Promotion Agency, Fujitsu \& NEC. 1999,2000. http: //www.wfmc.org/

[18] The Workflow Management Coalition Specification, Workflow Management Coalition Interface 1: Process Definition Interchange Process Model, Document Number WfMC TC-1016-P, Document Status-Version 1.1, (Official release), Issued on October 29, 1999, Author: Work Group 1, The Workflow Management Coalition-Confidential, 1994-1999 The Workflow Management Coalition. http: //www.wfmc.org/

[19] ebXML Requirements Specification, Version 1.06, ebXML Requirements Team, May 8 , 2001 http: //www.ebxml.org/

[20] The Workflow Reference Model; Workflow Management Coalition, Document Number TC00-1003, Document Status-Issue 1.1, 19-Jan-95, David Hollingsworth, http://www.eworkflow.org!

[21] T Jepsen, SOAP Cleans up Interoperability Problems on the Web, IT Professional, 2001, January-February, pp. 2 - 55.

[22] World Wide Web Consortium: simple Object Access Protocol (SOAP) v1.1, W3C Note, http: //www.w3c.org/TR/soap, May 2001.

[23] User's Guide, Information Technology, Technical Dictionary, Issue 1.0, 11 January 2000, RosetiaNet, http://rosettanet.org/

[24] Site Sections about RosettaNet Home, http: //rosettanet.org/Rosettanet/Rooms/DisplayPages/LayoutInitial, Marts 2002

[25] RosettaNet Dictionaries, Marts 2002, http: //rosettanet.org/

[26] Overview, Clusters, Segments and PIPs, RosettaNet, Issue 01.01.00, 12 September 2000, http: //rosettanet.org/ 
[27] PIP ${ }^{\circledR}$ Specification, Cluster 3: Order Management, Segment A: Quote \& Order Entry, PIP3A4: Request Purchase Order, Validated 02.0.00, 6 December 2001, PIP ${ }^{\circledR}$ Version Identifier: V02.00. http: //rosettanet.org/

[28] RosettaNet Implementation Framework, (RNIF) 2.0, Benefits of RNIF 2.0 and Comparison to RNIF 1.1, Prasad Yendluri, February 2000. http: //rosettanet.org/

[29] RosettaNet Implementation Framework: Core Specification, Version: Validated 02.00.00, 13 July 2001.http: //www.rosettanet.org/

[30] ebXML Technical Architecture Team. ebxml Technical Architecture Specification v1.0.4, http: //www.ebxml.org/specs/ebTA.doc 